\title{
HUMANIZAÇÃO DA ASSISTÊNCIA DE ENFERMAGEM FRENTEAO PACIENTE ONCOLÓGICO PEDIÁTRICO
}

\author{
HUMANIZATION OF NURSING CARE FOR PEDIATRIC \\ ONCOLOGICAL PATIENTS
}

\author{
Jozilane Pereira Soares ${ }^{1}$ \\ Jéssica Pereira Soares Alves ${ }^{2}$ \\ Edilson Salvador da Silva ${ }^{3}$ \\ Raquel de Negreiros Moreira Silva ${ }^{4}$
}

RESUMO: OBJETIVO: Identificar os benefícios da assistência humanizada na oncologia pediátrica descritas na literatura científica. METODOLOGIA: Trata-se de uma revisão integrativa da literatura, realizada nas bases de dados: LILACS e SciELO, a busca foi realizada no idiomaportuguês, considerando artigos publicados entre o período de 2011 a 2019, para tanto foram utilizados os descritores: humanização, assistência de enfermagem e oncologia pediátrica. Foram encontrados 82 artigos no LILACS e 34 no SCIELO, foi realizada a exclusão dos repetidos, o que resultou em nove artigos. RESULTADOS: Entre os estudos selecionados, observou-se que os fatores mais prevalentes relacionados aos benefícios da assistência humanizada na oncologia pediátrica foram: i) valorização do vínculo de confiança; ii) comunicação; iii) ter empatia, escuta atenta e respeito a individualidade. CONSIDERAÇÕES FINAIS: A humanização da assistência de enfermagem prestada à criança oncológica representa uma ferramenta vital para a recuperação do paciente, minimizando os traumas e os impactos negativos resultantes da doença.

Palavras chave: Enfermagem Oncológica. Humanização da assistência. Pediatria.

\footnotetext{
1 Graduanda do curso de Bacharelado em Enfermagem. União de Ensino Superior de Campina Grande -UNESC Faculdades. E-mail: enf.jozilaneps@gmail.com.

${ }^{2}$ Graduanda do curso de Bacharelado em Enfermagem. União de Ensino Superior de Campina Grande -UNESC Faculdades. E-mail: enf.jozilaneps@gmail.com.

3 Graduanda do curso de Bacharelado em Enfermagem. União de Ensino Superior de Campina Grande - UNESC Faculdades. E-mail: jessica_p.s.a@hotmail.com.

${ }^{4}$ Docente do Curso de Bacharelado em Enfermagem da União de Ensino Superior em Campina Grande - UNESC Faculdades. Enfermeira (Bacharelado e Licenciatura-UEPB). Mestre em Modelos de Decisão e Saúde (UFPB). E-mail: kel.dudunana@gmail.com.
} 
ABSTRACT: OBJECTIVE: To identify the benefits of humanized care in pediatric oncology described in the scientific literature. METHODOLOGY: It is an integrative review of the literature, carried out in the databases: LILACS and SciELO, the search was performed in Portuguese language, considering articles published between the period from 2011 to 2019, for which the descriptors were used: humanization, nursing care and pediatric oncology. We found 82 articles in LILACS and 34 in SCIELO, the exclusion of the repeated ones was performed, which resulted in 9 articles. RESULTS: Among the selected studies, it was observed that placing oneself in the other's place and offering supports beyond cancer-based techniques, including being oncological as an individual, being empathetic and strengthening bonds of love and trust makes the more humane nursing care. FINAL CONSIDERATIONS: The humanization of nursing care provided to the oncological child represents a vital tool for patient recovery, minimizing trauma and negative impacts resulting from the disease.

Keywords: Humanization of Assistance. Oncology Nursing. Pediatrics. 


\section{INTRODUÇÃO}

O câncer refere-se a um conjunto de mais de 100 doenças que têm em comum a proliferação descontrolada de células anormais que invadem tecidos e órgãos, podendo espalhar-se rapidamente para outras áreas do corpo. Os diferentes tipos de câncer se diversificam quanto à velocidade de mutilação das células e a capacidade de invadir tecidos e órgãos vizinhos ou distantes, classificado como metástase (INCA, 2019c).

Diferentemente do câncer do adulto, o câncer infanto-juvenil (entre 0 e 19 anos) geralmente acomete as células do sistema sanguíneo, os tecidos da sustentação e apresentam melhor resposta ao tratamento atual. Os tumores mais frequentes na infância e na adolescência são as leucemias, os que atingem o sistema nervoso central e os linfomas (INCA, 2019a).

Para cada ano do biênio (2018-2019) estima-se que no Brasil ocorrerá 420 mil novosde câncer. Como observado nos Registros de Câncer de Base Populacional, o percentual mediano dos tumores infanto-juvenis é de $3 \%$, deduzindo assim que ocorrerão 12.500 casosnovos de câncer em crianças e adolescente, destacando-se as Regiões Sudeste e Nordeste as que apresentarão maiores números de casos novos, 5.300 e 2.900, respectivamente (INCA, 2019b).

O câncer infantil provoca uma série de alterações na capacidade funcional, física, mental e psicológica. Essas mudanças resultam em um grande desafio para a criança oncológica, a família e os profissionais envolvidos no tratamento. Para minimizar os impactos causados pela doença, a assistência de enfermagem deve ser pautada nos princípios éticos, legais e humanísticos voltados para a criança e a família resultando em um cuidado acolhedor e eficaz (NEVES; MENDES; SANTOS, 2017).

Sentimentos como confiança e amizade devem estar presentes no cuidado à criança oncológica tendo em vista a redução do estresse e angústia presente nesse âmbito. A base de confiança sucederá por meio da comunicação verbal e não verbal, 
durante o contato paciente- enfermeiro, originando assim o cuidado sistematizado integral do ser (VIEIRA; CASTRO; COUTINHO, 2016).

Dentre as atribuições da enfermagem, voltada ao amparo paliativo do paciente oncológico pediátrico, destaca-se a conduta humanista, processo terapêutico centrado nos valores humanitários, estabelecendo uma relação interpessoal entre o infante oncológico e oenfermeiro (DELFINO et al., 2018).

A humanização da assistência à criança hospitalizada deve ser compreendida não sóna perspectiva dos cuidados e tecnologias disponibilizadas pelos profissionais e serviços desaúde, mas sim ao que se preconiza na atualidade, precedidos pelos familiares da criança, motivados pelas ações e atitudes seguindo os princípios éticos, humanístico, social e holístico(PEREIRA et al., 2018).

A temática da humanização na assistência de enfermagem prestada às crianças com câncer se faz necessária tendo em vista as particularidades de cada indivíduo, os efeitos colaterais, variações de sentimentos e impactos causados na vida da criança após ser diagnosticada com câncer. Diante disso, esse estudo temse como objetivo identificar os benefícios da assistência humanizada na oncologia pediátrica descritas na literatura científica.

\section{METODOLOGIA}

Trata-se de uma revisão da literatura onde seguiram-se as seis etapas proposta por Mendes, Silveira e Galvão (2008): identificação do tema; estabelecimento de critérios de inclusão e exclusão dos estudos, definição das informações a serem extraídas dos estudos selecionados; avaliação dos estudos incluídos na revisão; interpretação dos resultados e apresentação da síntese de conhecimento.

A identificação dos artigos de interesse foi realizada em maio de 2019, utilizando-se as bases de dados Scientific Electronic Library Online (SciELO) e da Literatura Latino- americana e do Caribe em Ciências da Saúde (LILACS). A busca 
foi realizada o idioma português, considerando artigos completos publicados entre o período de 2011 a 2019.

Nesta perspectiva foi elaborada a pergunta norteadora: Quais os benefícios da assistência humanizada de enfermagem na oncologia pediátrica?

Utilizou-se os seguintes Descritores em Ciência da Saúde (DeCS): "Humanização", "Assistência de Enfermagem" e "Oncologia Pediátrica", todos combinados entre si com a utilização dos operadores booleanos AND e OR.

Como critérios de inclusão no estudo, foram considerados artigos que tratassem do tema em estudo, publicados no período estabelecido, disponíveis gratuitamente e escritos na língua portuguesa, e foram excluídos os artigos que não atendiam aos critérios acima descritos.

\section{RESULTADOS}

No SciELO obteve-se 34 artigos, mas, destes, apenas cinco foram selecionados por atenderem aos critérios de inclusão e exclusão estabelecidos. No LILACS, obteve-se 82 publicações, realizou-se a leitura dos títulos e resumos com o objetivo de selecionar de acordo com os critérios de inclusão e exclusão, o que resultou na seleção de seis artigos. Dos 11 artigos selecionados foi realizado a exclusão dos repetidos, o que resultou nove artigos.

Para relacionar os artigos que compõem esta revisão, foi elaborado um quadro sinóptico para a coleta dos dados, contendo os seguintes componentes: autores/ano de publicação, título, tipo de estudo/número de participantes, objetivos e principais resultados (Quadro 1).

Entre os estudos selecionados, observou-se que os fatores mais prevalentes relacionados aos benefícios da assistência humanizada na oncologia pediátrica foram:

i) valorização do vínculo de confiança;

ii) comunicação;

iii) ter empatia, escuta atenta e respeito a individualidade. 
Quadro 1. Descrição dos artigos selecionados segundo/ano de publicação, título, tipo deestudo/número de participantes, objetivo e principais resultados.

\begin{tabular}{|c|c|c|c|c|}
\hline $\begin{array}{l}\text { AUTORESI } \\
\text { ANODE } \\
\text { PUBLICAÇÃO }\end{array}$ & $\begin{array}{l}\text { TíTULO DO } \\
\text { ARTIGO }\end{array}$ & $\begin{array}{c}\text { TIPO DO ESTUDOI } \\
\text { NÚMERO DE } \\
\text { PARTICIPANTES }\end{array}$ & OBJETIVO & $\begin{array}{l}\text { PRINCIPAIS } \\
\text { RESULTADOS }\end{array}$ \\
\hline $\begin{array}{l}\text { CAIRES et a } \\
2018\end{array}$ & $\begin{array}{ll}\text { Recidiva } & \\
\text { Oncológica: } & \\
\text { Olhares dos } \\
\text { Profissionais } & \\
\text { Hospitalares } & \\
\text { sobre } & \text { as } \\
\text { Dificuldades } & \text { do } \\
\text { Paciente } & \\
\text { Pediátrico } & \end{array}$ & $\begin{array}{l}\text { Estudo } \\
\text { transversal/17 } \\
\text { profissionais das } \\
\text { áreas de saúde, } \\
\text { educação, serviço } \\
\text { social e psicologia }\end{array}$ & $\begin{array}{|lr|}\text { Auscultar } & \text { as } \\
\text { percepções } & \text { dos } \\
\text { profissionais } & \text { de } \\
\text { oncologia } & \text { pediátrica } \\
\text { quanto às } & \text { principais } \\
\text { dificuldades } & \text { vividas } \\
\text { pelas } & \\
\text { crianças/adolescente } \\
\text { s com doença } \\
\text { oncológica durante a } \\
\text { recidiva da doença. }\end{array}$ & $\begin{array}{l}\text { Os resultados do estudo } \\
\text { apontam que o modo } \\
\text { direto de auscultar as } \\
\text { experiências vivenciadas } \\
\text { pelas crianças durante a } \\
\text { recidiva, favorece o } \\
\text { desenho er a } \\
\text { implementação de de } \\
\text { intervenções psicológicas } \\
\text { mais eficazes. }\end{array}$ \\
\hline $\begin{array}{l}\text { SILVA } \\
2018\end{array}$ & $\begin{array}{l}\text { Significado do } \\
\text { cuidar e seus } \\
\text { sentimentos para } \\
\text { equipe de } \\
\text { enfermagem } \\
\text { diante da criança } \\
\text { em tratamento } \\
\text { oncológico }\end{array}$ & $\begin{array}{l}\text { Estudo transvers } \\
10 \text { profissionais } \\
\text { enfermagem }\end{array}$ & $\begin{array}{|lr|}\text { O objetivo } & \text { desse } \\
\text { estudo } & \text { foi } \\
\text { compreender } & \text { os } \\
\text { significados } & \text { e } \\
\text { sentimentos } & \\
\text { manifestados } & \text { pela } \\
\text { equipe } & \text { de } \\
\text { enfermagem } & \text { durante } \\
\text { o cuidar num serviço } \\
\text { de } & \text { oncologia } \\
\text { pediátrica. } & \\
\end{array}$ & $\begin{array}{l}\text { Os resultados do estudo } \\
\text { apontam que a busca } \\
\text { pela humanização do } \\
\text { cuidado torna a } \\
\text { internação da criança } \\
\text { menos traumatizante e } \\
\text { abrange o conforto } \\
\text { e o cuidado para todos } \\
\text { os envolvidos. Tendo } \\
\text { como critérios: } \\
\text { cuidado, conforto e } \\
\text { humanização; }\end{array}$ \\
\hline $\begin{array}{l}\text { PEITER et a } \\
2016\end{array}$ & $\begin{array}{lr}\text { Gestão } & \text { do } \\
\text { cuidado } & \text { de } \\
\text { enfermagem } & \text { ao } \\
\text { paciente } & \\
\text { oncológico num } \\
\text { hospital geral: } \\
\text { umar Teoria } \\
\text { Fundamentada } \\
\text { dos Dados }\end{array}$ & $\begin{array}{l}\text { Estudo transversal/ } \\
14 \text { profissionais } \\
\text { enfermeiros, } \\
\text { técnicos } \\
\text { enfermagem, } \\
\text { nutricionista, } \\
\text { psicóloga e } \\
\text { familiar). }\end{array}$ & 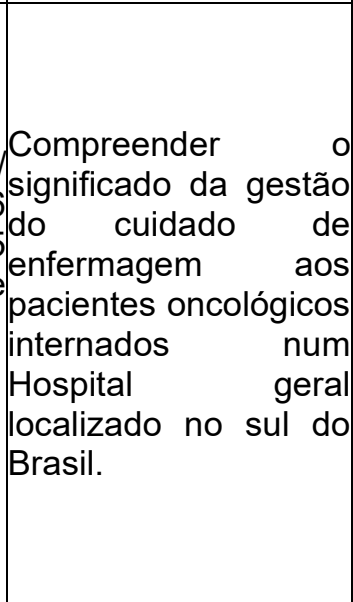 & $\begin{array}{l}\text { O laço de confiança por } \\
\text { meio do diálogo } \\
\text { possibilitou que r os } \\
\text { pacientes falassem de } \\
\text { seus }\end{array}$ \\
\hline
\end{tabular}




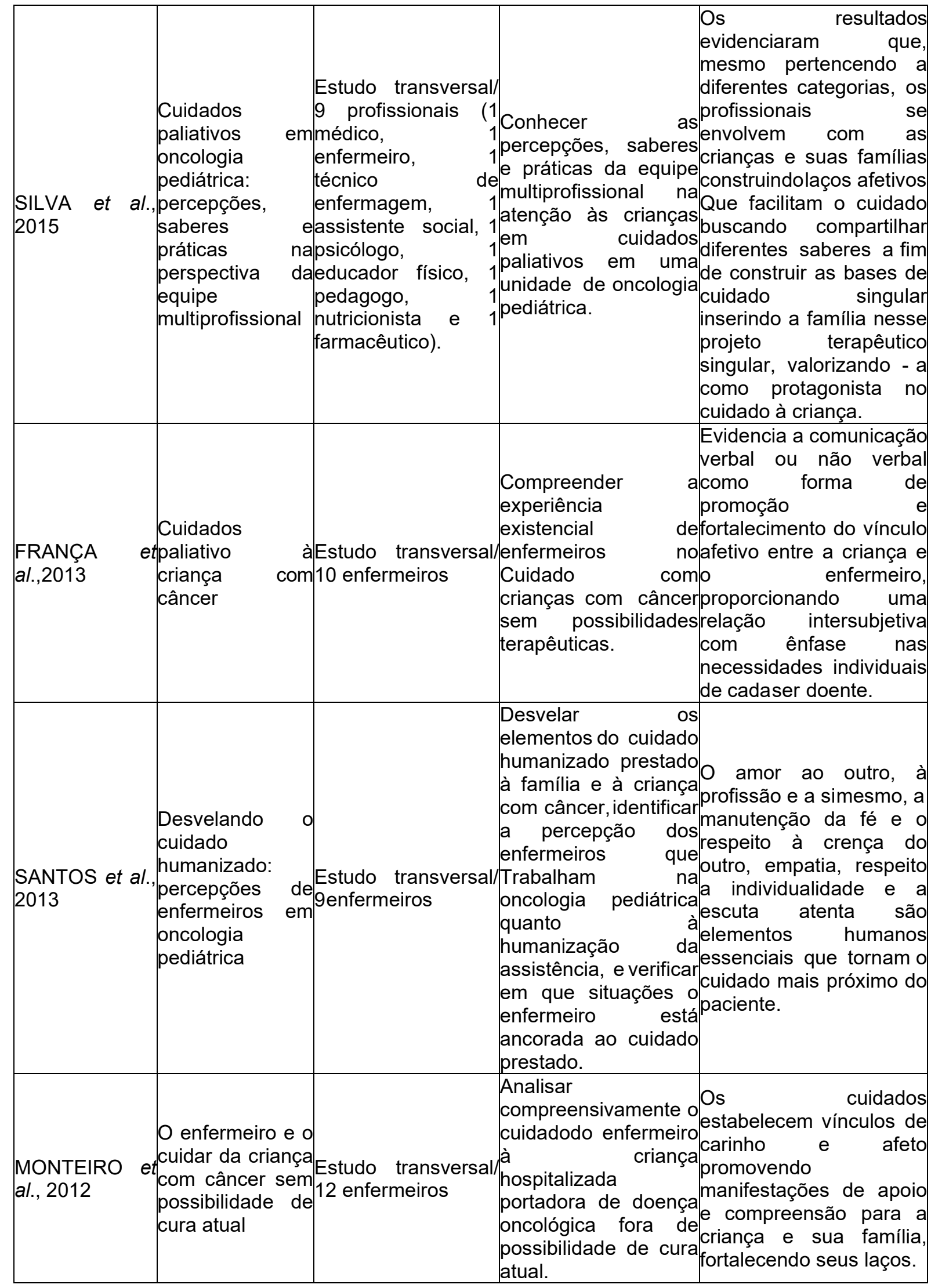




\begin{tabular}{|c|c|c|}
\hline $\begin{array}{l}\text { AMADOR et al. } \\
2011\end{array}$ & $\left|\begin{array}{lr}\text { Concepção } & \text { dos } \\
\text { enfermeiros } & \\
\text { acerca } & \text { da } \\
\text { capacitação } & \text { no } \\
\text { cuidado } & \text { Estudo transvers } \\
\text { criança } & \text { com } \\
\text { câncer } & \end{array}\right| \begin{array}{l}\text { Eenfermeiras } \\
\end{array}$ & 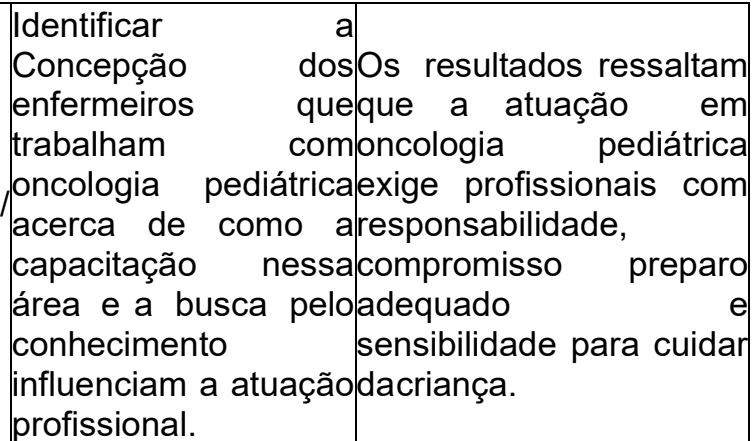 \\
\hline $\begin{array}{l}\text { MARANHÃO } \\
\text { al., } 2011\end{array}$ & 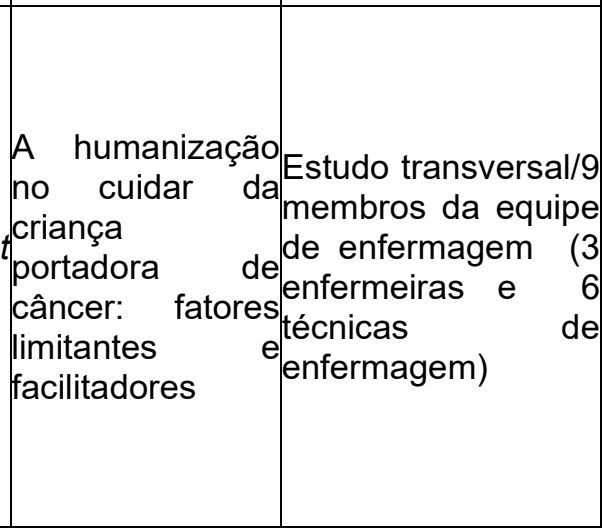 & 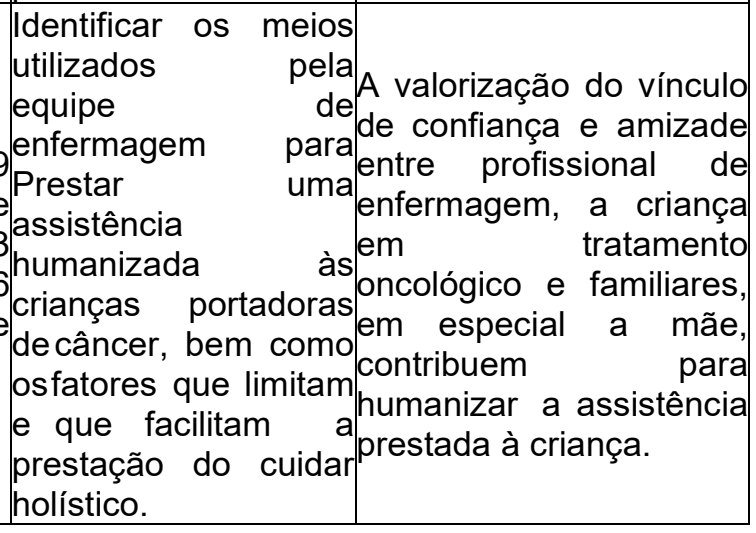 \\
\hline
\end{tabular}

Fonte: Pesquisa, 2019.

\section{DISCUSSÃO}

De acordo com os estudos selecionados, o estabelecimento do laço de confiança possibilita que os pacientes verbalizem sobre seus medos e encoraja-os a expressar suas dúvidas, anseios e expectativas, estabelecendo uma relação de solidariedade e apoio entre profissional/paciente diante do enfrentamento da doença (FRANÇA; COSTA; NÓBREGA,2013).

A valorização desse vínculo de confiança e amizade entre o profissional de enfermagem e a criança em tratamento oncológico permite uma assistência humanizada compreendendo o paciente enquanto ser humano, a fim de reduzir o estresse e a angústia desencadeados pela realidade da condição clínica (MARANHÃO et al., 2011).

Esse vínculo não deve limitar-se apenas entre o enfermeiro e a criança, mas também com seus familiares, em especial a mãe, peça-chave facilitadora do 
processo do cuidar, ampliando melhor a compreensão do atendimento profissional para com a criança de maneira integral (MARANHÃO et al., 2011).

No tratamento oncológico é primordial que o profissional de enfermagem perceba, compreenda e empregue adequadamente a comunicação verbal e nãoverbal. A comunicação não-verbal permite a percepção e compreensão dos sentimentos, dúvidas, angústias do paciente, entendimento e percepção de gestos e olhares expressados pela criança (ARAÚJO;SILVA, 2012).

A comunicação se faz necessária, pois favorece a autonomia, manutenção da capacidade de expressar-se, ativa a participação social e melhora a qualidade de vida da criança com câncer (ARAÚJO et al., 2018).

Tendo em vista a humanização, o enfermeiro deve realizar um cuidado focado na proteção, na solicitude, na escuta, no restabelecimento da força, principalmente em momentos de um desfecho inevitável, deixando o paciente confortável mesmo sem estar saudável. Esse cuidado proporciona o alívio da dor e do sofrimento causados pela doença ou até mesmo devido ao seu tratamento (MONTEIRO; RODRIGUES; PACHECO, 2012).

Desta forma, o cuidado humanizado focado no conforto exige do profissional tempo, atenção, sensibilidade, solidariedade e disponibilidade para entender e compreender as necessidades da criança (MONTEIRO; RODRIGUES; PACHECO, 2012).

Almejando uma assistência humanizada, o cuidar da criança durante o tratamento oncológico, os integrantes da equipe de enfermagem envolvem esse cuidado com amor, confiança, amizade e escuta, não se limitando apenas ao tratamento medicamentoso. Essa visão do profissional resulta em qualidade no atendimento prestado à criança e aos seus familiares (SILVA et al.,2018).

A enfermagem deve realizar seu trabalho de forma acolhedora, segura e humanística garantindo à criança com câncer qualificação da assistência prestada, apoio e conforto à família para proporcionar alívio dos impactos causados pelo tratamento (NEVES; MENDES;SANTOS, 2017).

É imprescindível que para uma assistência humanizada e de qualidade ao paciente oncológico, o enfermeiro deve agir com empatia, analisando e compreendendo o mundo característico da criança para aprimorar a percepção e a 
relação. Esse profissional tem como função mensurar o quanto a criança e sua família são atingidos pelo abalo do diagnóstico, tratamento agressivo e regresso à vida após o tratamento. Sendo assim, a conduta do enfermeiro exprime promoção de aconchego e bem-estar, por meio de carinho e atenção proporcionando suporte emocional e espiritual (PIRES et al., 2017).

Desta forma, o colocar-se no lugar do outro e oferecer suportes além das técnicas voltadas para o tratamento do câncer, compreendendo a criança com câncer como um ser individual, ter uma escuta mais apurada e fortalecer laços de amor, confiança e amizade para com esse ser e sua família, são elementos que favorecem à humanização da assistência de enfermagem.

\section{CONSIDERAÇÕES FINAIS}

O presente estudo possibilitou perceber que a humanização da assistência de enfermagem prestada à criança oncológica deve ser compreendida além da perspectiva dos cuidados e tecnologias disponibilizadas pelo serviço de saúde.

O câncer compromete não somente o meio físico da criança como também o emocional dela e de toda a família, por esta razão, a equipe de saúde, principalmente o enfermeiro se transfigura em ser fundamental para a assistência humanizada.

A humanização do cuidar representa uma ferramenta vital para a recuperação do paciente, minimizando os traumas e os impactos negativos resultantes da doença. Por essa razão, se faz necessário que o profissional de enfermagem desenvolva técnicas que incluam vínculo de amizade, confiança, comunicação e afeto voltados para a criança e a sua família, promovendo assim, bem-estar e conforto de todos os envolvidos. 


\section{REFERÊNCIAS BIBLIOGRÁFICAS}

AMADOR, D. D; GOMES, I. P; COUTINHO, S. E. D; COSTA, T. N. A; COLLET, N. Concepção dos enfermeiros acerca da capacitação no cuidado à criança com câncer. Texto Contexto, v. 20, n. 1, p. 94-101, 2011. Disponível em: http://dx.doi.org/10.1590/S010407072011000100011. Acesso em 20 mai. 2019.

ARAUJO, G. S; PAULO, A.M. F; NETA, H. H. S; COSTA, L. B; SANTOS, S. N. S. F; LIMA, I. L. B. Benefícios da tecnologia de comunicação aumentativa e alternativa em pacientes oncológicos. Revista Saúde e Ciência online, v.7, n. 2, p.502, 2018. Disponível em: http://www.ufcg.edu.br/revistasaudeeciencia/index.php/RSC- UFCG/article/view/625/362. Acesso em: 22 mai. 2019.

ARAÚJO, M. M. T; SILVA, M. J. P. Estratégias de comunicação utilizadas por profissionais de saúde na atenção à pacientes sob cuidados paliativos. Revista da Escola de Enfermagem da USP. São Paulo, v.46, n. 3, p. 626-632, 2012. Disponível em: http://www.periodicos.usp.br/reeusp/article/view/40990/44516. Acesso em: 22 mai. 2019.

CAIRES, S; MACHADO, M; ANTUNES, M. C; MELO, A. S. M. Recidiva Oncológica: Olhares dos Profissionais Hospitalares sobre as Dificuldades do Paciente Pediátrico. Psico- USF, Bragança Paulista, v. 23, n. 2, p. 333-345, 2018. Disponível em: http://dx.doi.org/10.1590/141382712018230212. Acesso em: 23 mai. 2019.

DELFINO, C. T. A; FERREIRA, W. F. S; OLIVEIRA, E.C; DUTRA, D.A. Câncer infantil: Atribuições da enfermagem em cuidado paliativo. Revista Saúde e Desenvolvimento. v. 12, n.10, p.496, 2018. Disponível em: https://www.uninter.com/revistasaude/index.php/saudeDesenvolvimento/article/view/866/4 97. Acesso em: 21 abr. 2019.

FRANÇA, J. R. F. S; COSTA, S. F. G; NÓBREGA, M. M. L; LOPES, M. E. L. Cuidados paliativos à criança com câncer. Revista Enfermagem UERJ. Rio de Janeiro, v.21, n. 2, p.779784, 2013. Disponível em: http://pesquisa.bvsalud.org/portal/resource/pt/lil-748570. Acesso em: 20 mai. 2019.

INCA, Instituto Nacional do Câncer. 2018. Disponível em: https://www.inca.gov.br/câncerinfantojuvenil. Acesso em: 25 abr. 2019.

INCA, Instituto Nacional do Câncer. 2018. Disponível em: www1.inca.gov.br/estimativa/2018/síntese-de-resultados-comentários.asp. Acesso em: 25 abr. 2019.

INCA, Instituto Nacional do Câncer. 2019. Disponível em: https://www.inca.gov.br/o que- ecancer. Acesso em: 25 abr. 2019.

MARANHÃO, T.A; MELO, B.M.S; VIEIRA, T.S; VELOSO, A.M.M.V; BATISTA, N.N.L.A.L. A humanização no cuidado da criança portadora de câncer: fatores limitantes e facilitadores. Journal of the Health Sciences Institute, Teresina- PI, v.29, n.2, p. 106-109,2011. Disponível em: $\quad$ https://www.unip.br/presencial/comunicacao/publicacoes/ics/edicoes/2011/02_abrjun/V29_n2_2011_p106-109.pdf. Acesso em: 20 mai. 2019.

MONTEIRO, A.C. M; RODRIGUES, B. M. R. D; PACHECO, S. T. A; PIMENTA, L. S. O enfermeiro e o cuidar da criança com câncer sem possibilidade de cura atual. Escola Anna Nery, v. 16, n. 4, p. 741-746, 2012. Disponível em: http://dx.doi.org/10.1590/S141481452012000400014. Acesso em: 22 mai. 2019. 
NEVES, J. N; MENDES, D. R. G; SANTOS, W. L. Enfermagem em oncologia pediátrica: Fatores de excelência na assistência integralizada. 2017. Disponível em: http://www.senaaires.com.br/wp-content/uploads/2017/05/ENFERMAGEM-EM- ONCOLOGIAPEDI\%C3\%81TRICA-FATORES-DE-EXCEL\%C3\%8ANCIA-NA-

INTEGRALIZADA.pdf. Acesso em: 23 mai. 2019.

ASSIST\%C3\%8ANCIA-

PEITER, C. C; CAMINHA, M.E.P; LANZONI, G. M. M; ERDMANN, A. L. Gestão do cuidado de enfermagem ao paciente oncológico num hospital geral: uma Teoria Fundamentada nos Dados. Revista de Enfermagem Referência, v. IV n. 11, p. 61-69, 2016. Disponível em: http://dx.doi.org.10.12707/RIV16044. Acesso em: 23 mai. 2019.

PEREIRA, C R; LIMA, K. G. J; RODRIGUES, M. T. M; DURÃES, P. J. A; NEVES, S. J.O; VIANA, $\mathrm{T}$. M, et al. A humanização da assistência de enfermagem à criança hospitalizada: uma revisão integrativa. Revista Intercâmbio. v. XI, p.081-082, 2018. Disponível em: http://www.intercambio.unimontes.br/index.php/intercambio/article/view/224. Acesso em: 28 abr. 2019.

PIRES, C. O. S. A. S; MACHADO, D.G; VARGAS, G.L; BORDIN, P. M. A; TAVARES, S. L. S. O papel do enfermeiro na oncologia pediátrica. URCAMP BAGÉ-RS, 2017. Disponível em: http://trabalhos.congrega.urcamp.edu.br/index.php/14mic/article/view/1733/1880. Acessoem: 23 mai. 2019.

SANTOS, M. R; SILVA, L; MISKO, M. D; POLES, K; BOUSSO, R.S. Desvelando o cuidado humanizado: percepções de enfermeiros em oncologia pediátrica. Texto contexto enfermagen. Florianópolis, v. 22, n. 3, p. 646-653, 2013. Disponível em: http://www.scielo.br/pdf/tce/v22n3/v22n3a10.pdf. Acesso em: 22 mai. 2019.

SILVA, A. F; ISSI, H. B; MOTTA, M. G.C; BOTENE, D. Z. A. Cuidados paliativos em oncologia pediátrica: percepções, saberes e práticas na perspectiva da equipe multiprofissional. Revista Gaúcha de Enfermagem, v. 36, n. 2, p.56-62, 2015. Disponível em: http://dx.doi.org/10.1590/1983-1447.2015.02.46299. Acesso em: 23 mai. 2019.

SILVA, C. M. N; SILVA, M. P. C; FERREIRA, D. O; AMARAL, J.B; GONÇALVES, J. R. L; CONTIM, D. Significado do cuidar e seus sentimentos para a equipe de enfermagem diante da criança em tratamento oncológico. Revista de Enfermagem e Atenção à Saúde [Online]. Uberaba. $\quad$ v.7, n. 2,p.83-94, $2018 . \quad$ Disponível em: http://seer.uftm.edu.br/revistaeletronica/index.php/enfer/article/viewFile/2355/pdf_1. Acesso em: 23 abr. 2019.

VIEIRA, A. M. P. E; CASTRO, D.L.; COUTINHO, M.S. Assistência de Enfermagem na Oncologia Pediátrica. Revista Eletrônica Atualiza Saude. Salvador. v.3, n.3, p.67-75. 2016. Disponível em: <https://atualizarevista.com.br/wp. Acesso em: 30 abr. 2019. 